\title{
The Spectral Sign: A Cybernetic Perspective on Digital Conversations
}

\author{
Marco Toledo Bastos \\ Universidade de São Paulo, Escola de Comunicações e Artes \\ Av. Prof. Lúcio Martins Rodrigues, 443 -Bloco A, sala 41 \\ Cidade Universitária, Butantã, São Paulo, 05508-020, Brazil \\ http://marco.toledobastos.com
}

\begin{abstract}
This paper discusses the idea of a spectral sign in relation to the concept of multilogue (Shank 1993) and crosstalk (Crystal 2001), a discussion largely based upon linguistic and semiotic contexts. In short, spectral sign is the outcome of an operation that corrupts the semiotic structure of a sign, replacing instead of adding units of meaning. From a linguistic point of view, the spectral sign relies on the effects of communication technologies that challenge the dyadic representation of a sign. Instead of relating to another sign to perform a paired circle, spectral sign connects a diversity of circles that are not immediately accessible in a semiotic context. From a cybernetic point of view, this interplay represents negative feedback between observer and observed and reveals digital meaning as being simultaneously two: one related to the source, and the other to the receiver.
\end{abstract}

\section{Introduction}

This paper discusses the idea of a spectral sign in relation to the concept of multilogue and crosstalk, a discussion largely based upon the linguistic and semiotic contexts. Duke (1974) first presented the term multilogue as a pattern of interaction pertaining to game experiences that described a many-to-many conversational thread. The concept was later explored by Shank (1993), who made a comparative analysis between Jakobson's linguistic model and the nature of interactions through social media and digital interactive tools. At this later revision, multilogue was depicted as a polylogue communication involving a 
linguistic variation that Shank described along a series of articles. However, the author refrained from offering a diagram for the multilogue communication, and this paper intends to fill this gap presenting a diagram of multilogues and the spectral signs. We expect this visual diagram to contribute to the debate on the interactive patterns of multilogue and crosstalk.

Spectral sign is a linguistic variation that relies on digital technologies and challenges Saussure's model of the sign as a self-contained dyad. From a cybernetic point of view, this interplay can be described as negative feedback between observer and observed, or as a series of interruptions between message and channel that is ultimately incompatible with sender-receiver communication framework (Shannon 1949). As we discuss in the second part of this paper, the signifying activity in multilogues not only relate to another sign, therefore performing single paired circles, but also to a diversity of circles that are not immediately accessible in the semiotic context. Signifiers are thus able to relate to other signifiers before conceiving a signification. From a Structuralist point of view, signs not only relate to a second sign in the same circle, but instead to another sign in a different circle, and continuously move along a path of crosstalk and spirals of meanings.

The breaking of a linguistic chain created by the spectral sign becomes clear when presented together with a corresponding diagram of denotation and connotation. While denotation refers to the literal meaning of a word, or the definition a dictionary offers, connotation refers to secondary associations that are carried with the word or the emotional suggestions related to that word. Denotative and connotative meanings coexist within most words, and it is possible to schematically present this coexistence in linguistic terms: while denotative signs trace a signified/signifier relation, connotative signs replicate this relationship inside the same signifier. That is, connotation unfolds the denotative signification toward a new sign-relation, thus recodifying the original meaning. Because of that, connotation is essentially an operation of meaning addition. It appends signifieds and signifiers to a given sign while preserving the sign as a selfcontained dyad. The dyadic arrangement is what guarantees the ambiguity conveyed by the messages, and therefore is necessary to puns, jokes and other colloquial forms of humor and crosstalk.

Spectral meaning interrupts this process of continuous addition of meanings. Instead of adding more meanings to the basic definition, spectral signs have their meanings supplanted to a new set of references. While crosstalking and connotation support relationships between signified and signifier already given in a sign, the spectral sign 
appends only signifiers — the word or the sound-image — to another signifier. As a result, it is not a development on the possibilities of signification, but instead a distortion or corruption of the original signification. This difference becomes more pronounced when comparing the adding of signified and signifier in connotative signs to the spectral, which only includes other signifiers in a series of degenerative chains. The structure of the degenerative chains and the particulars of this communication pattern are discussed in the last section of this paper.

\section{Spectral Sign}

The concept of spectral sign is the result of a number of studies of chat rooms during the late 1990's. The literature tried to grasp the unpredictable nature of meaning within chat rooms, identifying features from synchronous and asynchronous messages and describing meaning generation as an operation not specifically given by context, so long as context is governed by linguistic rules and cultural conventions. Phrases or words would suddenly crack a point of disruption when social interactions follow complex contagion or viral propagation of ideas and trends. The effect required a state of extended attention from users attending the communication channel and it was normally triggered by a random word. Given that any keyword in a given thread can be potentially disruptive, the social effect was not the outcome of words, but of the continuous variation of meaning provided by words.

The striking characteristic of chat rooms was its ability to burst interactions because of misunderstandings or communicational setbacks provided by the lack of visual clues in the medium. The concept of multilogue presented by Shank (1993) addressed the problem of meaning in digital environments as a linguistic and communication issue. The rapid rise of social networks such as Twitter or Facebook framed again the problem during the 2000's and suggested that chronic misunderstanding in digital platforms was not restricted to synchronous interfaces. The comment areas on blogs also experienced in the early years of 2000's a similar pattern of miscommunication, thus supporting Shank's hypothesis that synchronous and asynchronous interaction followed comparable paths in meaning processing.

The core of the problem lies less in the differences between synchronous and asynchronous interaction and more in the differences between intended and automatic messages. The limits of interaction and the random results provided by smart software 
such as chat-boxes or interactive bots were tested when digital services were first made available, and continued to be tested during the 1990's and 2000's. These autonomous systems of make believe interaction were progressively incorporated to the communication infrastructure, with indirect but no less significant effects to social meaning. Even when interaction was limited, it was still ruled by the digital ecosystem that governed digital information.

Digital communication has changed the interactive experience to the point of affecting linguistic structures used to convey such experiences. Crystal (2001) calls netspeak the new language of information communication found on the internet and chat rooms. If speech is defined by time-bound, spontaneous, socially interactive, loosely structured, immediately revisable and prosodically rich utterances, and writing is defined by spacebound, contrived, visually decontextualized, factually communicative, elaborately structured, repeatedly revisable and graphically rich forms, then netspeak would rely on characteristics belonging to both sides of the speech/writing divide.

Digital media presents a myriad of channels that both facilitate and constrain human ability to communicate in ways other than those found in other semiotic situations. Procedures and practices associated with spoken and written language no longer limit the linguistic horizon of a digital landscape where the time frame of the participants hardly coincides. Turn-taking, once the fundamental parameter to understand other peers, no longer governs the interaction. The lack of interaction control deteriorates with the increasing number of overlapping messages that a screen can display synchronously. Even though interaction increases together with the number of participants, understanding of the messages actually decreases proportionally. Generally, the possibilities of misunderstanding and confusion in digital communication are enormous (Crystal 2001).

Crystal concludes his investigation claiming that digital language is not exactly an "emerging language centaur" - part speech, part writing. It would be more than just a hybrid of speech and writing, or the result of contact between two long-standing media. Digital texts of any kind, says Crystal (2001), are simply not the same as others kinds of texts. They are rather a genuine "third medium." Still according to Crystal (2001), netspeaking is an original phenomenon that is neither spoken writing nor written speech, but something different from both writing and speech that can be addressed as a forth medium. This is what allowed the British linguist to speak in terms of spoken language, written language, sign language and computer-mediated language. This fourth medium sheds light onto the idea of multilogues and the spectral sign. 
The term multilogue was first presented by Duke (1974) as a pattern of interaction developed in games that described a many-to-many conversational dialogue. The concept was later explored by Shank (1993), who made a comparative analysis between Jakobson's linguistic model and the nature of interactions through social media and digital interactive tools. Multilogue is a polylogue communication involving linguistic variations that Shank described through a series of articles. The description of the interactions generated by multilogues and crosstalk is analogous to the spectral sign, as both present a linguistic variation produced by digital technology that differs from the associative meanings of words and phrases. The differences are subtle, but very important. While crosstalk comprises of an oscillatory level of noise and understanding, the spectral sign could be described as multiple second-order observations over a steady background noise.

The spectral sign does not depend on sender-receiver entities, and the text itself is detached from necessities of expression. The text is understood as interplay of signs regulated less by the content it signifies than by the nature of the signifier. Following this distinction, Terranova (2004) criticized both Shannon' information theory and semiotic approaches that assigned necessary significations to signs. According to the author, Shannon's linear conception of communication, which implies a circuit linking senders to receivers through a channel, was turned upside down when communication turned nonlinearized, without simple causal relationships or proportions between input and output. When information is no longer transmitted from point A to point $\mathrm{B}$ via a given channel, but rather starts jumping around and multiplying from channel to channel, from network to network, the very concept of channel is opened up onto an informational milieu.

This process is carried out daily in social networks such as Twitter, the micro-blogging service free of charge that enables 140 characters messages known as tweets. According to Rothstein (2009), the usual tweet is not properly a message, but a compound of codes and tags that organize the delivered data. For instance, "TheGlobeTripper RT @BradCrooks Google Squared... new user interface coming soon http://cli.gs/YzVBgN \#google \#search" has only 48 characters that are actually readable text. The rest represents hyperlinks, short-hand, tags and citations. It is also a reply to a previously sent tweet, which means there is no original content in the message. It is actually an index that points in a direction through the network. The message does not deliver actual content, but rather links that drive the reader through the network. The user is a node inside the network whose function is to redistribute information. 
Not only are tweets not proper messages, but also Twitter is not properly a channel (Terranova 2004). Twitter interface has taken particular aspects of blogs (the networked re-direction) and chats (the crosstalk) to deliver both features in a 140 characters limited message. It presents the indexing quality of the internet, the hyperlink, combined with the interactive and dynamic features of chatting. As a web of index fingers, it points from one spot on the net to another. If each Twitter user could be considered as a tag itself - the tag being the combination of its interests and characteristics - what the system performs is an operation of tagging the entire internet (Rothstein 2009).

Terranova (2004) also argues that postmodern theory captured and anticipated this development (the primacy of information networks over networks of meaning) when it described the culture of late capitalism as a culture of "floating signifiers," that is, a culture of signs that have lost their anchorage in networks of signification. According to the author, the image of signs that refer only to other signs in a relationship of preemptive causality is connected to the linear outcome of the commodification of culture. The postmodern description, in short, depicts a semiotic world out of control. The multilogue is not only a pattern of interaction, but also a linguistic operation prior to the communication level.

\section{Notations}

Flusser (2002) explored the linguistic functions of denotation and connotation as a relation of symbol and meaning that oscillates between two extremes. The "denoting" function (denotation) depicts, on one extreme, codes in which each symbol of the repertoire has a single meaning in the universe, and each meaning in the universe is represented in the code by a single meaning (a bi-univocal relationship between the code and its universe). "Connotating," on the other extreme, depicts a function in which each symbol of the repertoire may have various meanings in the universe, and each meaning in the universe may be represented in the repertoire by various symbols. In some cases, "denoting" refers to formal logic, while the connotating relates to the code of dream symbology. Most codes are mixed and have in their repertoire both denoting and connotating symbols, and as a result they convey both meanings by the use of a diversity of symbols.

The more denotating a code is, the more it was established conventionally. The more connotating it is, the more it is unstable (scientific codes, for instance, try to eliminate all connotations). Flusser (2002) claimed that behind this interpretation is the Cartesian 
ideal of a "clear and distinct message," like the formal model provided by Shannon's theory. Yet, there is a vast amount of highly conventionalized codes that aim specifically at the connotation, offering a range of significance that is concealed from the clear denoting message. As a result, connotation codes can transmit more "significant" messages (they capture their universes as a whole), even though the messages are prone to possible misunderstandings. According to Flusser, denoting messages are "closed" — they allow the receivers to have only one interpretation. Connotating messages, on the other hand, are "open" - they open to the receivers a parameter of interpretation. Selection between denotation and connotation, provided by a range of symbol and meanings, is usually set by a plan that aims at certain behavior patterns of the receivers. These two functions of communication can be used to describe the linguistic coding of the spectral sign.

When Shank (1993) presented the concept of multilogue, he was trying to analyze the crosstalking attributes of synchronous interaction. Multilogues share a great deal of common traits with crosstalk, but the differences are also significant. While the later tend to handle denotative and connotative meanings toward the creation of a comic scene, the former leans toward the creation of nonsense. Crosstalks add different meanings to a given sign, thus producing unexpected units of meaning and often producing nonsensical conversations. The spectral sign, on the other hand, replaces prior levels of significance and cannot be described as a self-contained dyad or by the linguistic notions of denotation and connotation.

For semiotics, denotation and connotation describe the relationships between the signifier and the signified, providing analytic distinctions in connection to these two types of signifieds. According to Silverman (1983), connotation is possible only in relation to a cultural code that provides a common framework to organize oppositions and functions. The common framework offers a horizon of meaning that aligns each term with a complex of symbolic attributes. Denotation, on the other hand, is described as the definitional or literal meaning of a sign provided by the framework of commonsense. The denotative meaning is what a dictionary provides or what viewers from any culture at any given time would say the image means (Panofsky 1982). Connotation, on the contrary, generally refers to socio-cultural and personal associations of the sign.

In other words, denotative meaning refers to a primary relationship between a given sign and a given object, while connotative meanings introduce a secondary notation (cum notatio) into the same relationship, thus widening the range of meanings. Connotation is related to significance and requires a comprehensive network of signs working together. 
It offers an alternative approach to infer the same object, echoing secondary meanings the object contains. In addition to that, the connotative operation cannot be separated from the object or its denotative meaning, as denotation is a prerequisite to connotation.

If denotation relies on a signifier and signified relationship, then connotation reproduces this relationship inside of a signifier, that is, it unfolds the original signifier into a new relationship that over-codifies the original meaning. According to Prieto (1966), connotation is an operation of adding signification that occurs when the signifier and signified of a sign become the signifier of an additional sign without taking away the preceding signified. In other words, connotation attaches supplementary denotations to signs as a layer cake of meanings. This regular addition of signifiers and signifieds does not change the self-contained dyadic structure of the sign, as there would be no ambiguity during crosstalk if the structure was changed.

Barthes (1957) seized upon Hjelmslev's notion of different orders of signification to describe connotation and denotation as levels of representation or levels of meaning. Denotation would stand for the first order of signification, a level in which the sign consists of a signifier and a signified. Connotation would stand for the second-order of signification, an operation that uses the denotative sign (signifier and signified) as signifier and attaches to it an additional signified. The spectral sign, however, does not add further meanings. Instead, it supersedes the original meaning and disconnects it from the former context. That is why an e-mail message can be misunderstood in different ways, detaching itself from the previous connotation and creating new and unpredictable meanings.

While denotation is associated with a sign and connotation is a result of associative phrasing, spectralization is situated at the level of the discourse and it disrupts the connotation/denotation balance. By replacing instead of adding more layers of meaning, it is fundamentally different from crosstalk or connotative phrasing. It does not result from the possibilities of signification. Instead, the spectral sign appends new significations to a given signifier and corrupts the original meaning. This difference becomes more apparent when we schematically compare denotation, connotation and spectrality. Figure 1 shows that while the former adds further signifiers and signified to a sign, the latter mostly refers to another meaning. Figure 2 shows the differences between linguistic and spectral signs. 


\section{Levels of Meaning}

\begin{tabular}{|c|c|}
\hline \multicolumn{2}{|c|}{ sign } \\
\hline signified & signifier \\
\hline
\end{tabular}

\begin{tabular}{|l|l|l|}
\hline \multicolumn{2}{|c|}{ sign } \\
\hline signified & \multicolumn{2}{|c|}{ signifier } \\
\hline & signified & signifier \\
\hline
\end{tabular}

Connotative

Meaning

\begin{tabular}{|c|c|c|}
\hline \multicolumn{2}{|c|}{ sign } & \multirow{2}{*}{$\begin{array}{l}\text { Spectra } \\
\text { Meanin }\end{array}$} \\
\hline signified & signifier & \\
\hline & signifier & signified \\
\hline signified & signifier & \\
\hline
\end{tabular}

Figure 1: Differences between Denotation, Connotation and Spectrality 


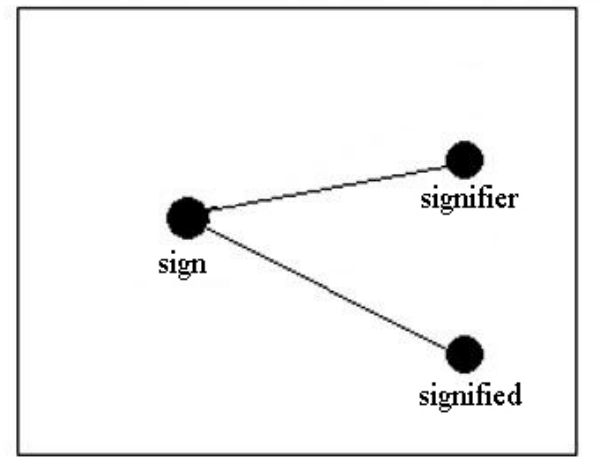

1. Diagram of linguistic sign

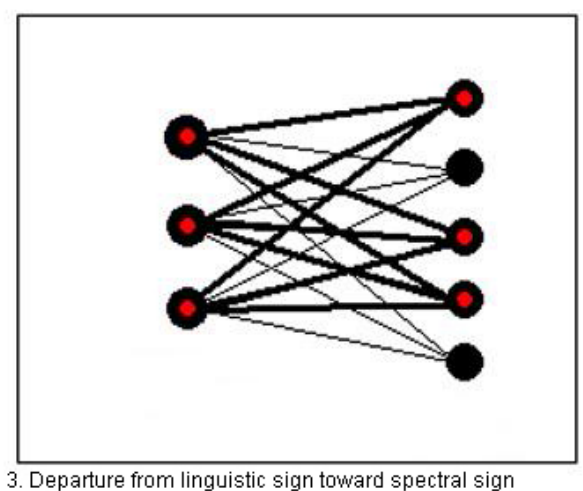

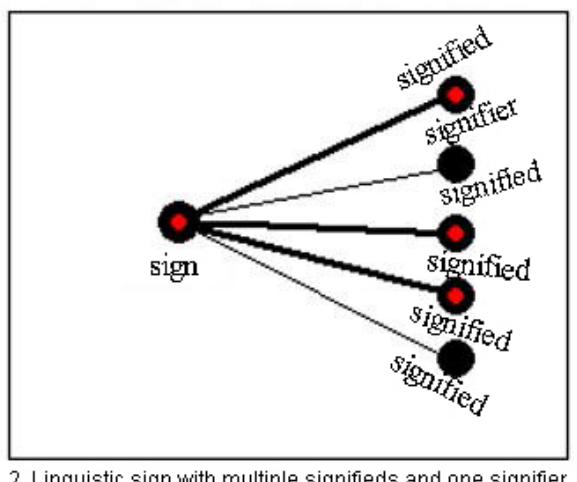

2. Linguistic sign with multiple signifieds and one signifier

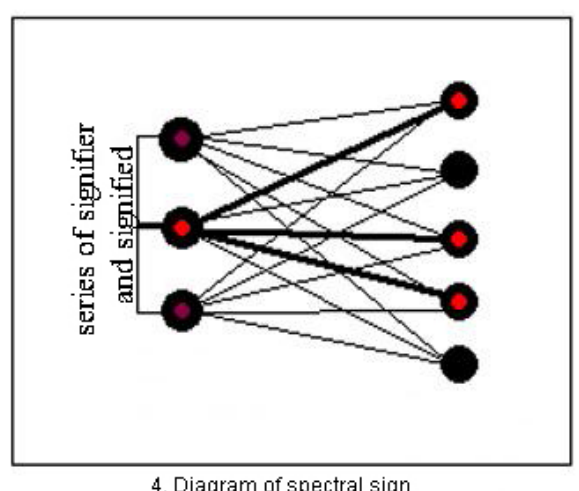

Figure 2: Phase diagrams from linguistic to spectral sign.

Shank' concept of multilogue was created to analyze the crosstalking attributes of online interaction. Shank based the concept on Jakobson's model of three basic types of conversation: the monologue, with one sender and one or multiple passive receivers; the dialogue, where sender and receiver take turns and; the discussion, where someone begins the conversation as a sender and multiple receivers take turns as a sender. Even though this model resembles the conversation on the internet, during the discussion the initial sender still retains control of the conversation. Similar to most academic settings where debate is encouraged, the professor is the initial sender who monitors and controls the discussion. The initial sender retains the position of control because the conversation depends upon the dyadic turn-taking, even with multiple sender-receiver combinations. 
But according to Shank (1993), the semiotic models of communication cannot target the experience of a conversation on digital networks. The linguistic models of lecture, dialogue and discussion cannot capture the dynamics of sign usage embodied in digital communication. Shank proposes the multilogue linguistic model in which there can be any number of players. The initial sender starts the thread and once it has been started, it is no longer under sender's control. The model is consistent with data exchange on the internet, where user interaction and data packaging are not dependent on turn taking. The fact a user follows up a given thread does not necessarily mean adhesion to the topic. What binds one interaction to another is not the content, but the serialized structure of connections that lacks turn taking.

The lack of context-dependency can be verified through the pursuit of meaning in digital interaction, which may lead to strange and unusual incidents. Shank (1993) tells an anecdote that took place on the Erl-l list of educational research. A member accidently sent a private message about the need to keep the refrigerator door shut to the entire list. Despite the fact that the user apologized immediately, many people seemingly failed to see the apology and took the message as a cryptic note about the state of educational research today. The metaphor continued expanding as people were considering the significance of the contents in the refrigerator, the fact that the door does not seal easily or the amount of energy required to keep the food cold. This story points out to specific hybridizations between circles of signs that were not predicted in Jakobson's model of conversation. It is not an operation in which signs change their semiotic matrices. Instead, it is the former semiotic matrix that is referring to an external semiotic matrix.

\section{Conclusion}

Aarseth (1998) argued that purely semiotic theories of computer-mediated phenomena are bound to fail in the analysis of the cybernetic sign. The reason is that semiotic theories are not concerned with the sign-production mechanism, without which the spectral sign cannot be properly understood. Aarseth's statement about semiotic theory not being well-equipped to describe ergodic modes of discourse can also be extended to the linguistics framework. According to Shank (1993), the linguistic models of lecture, dialogue, discussion and text (the written model of communication) do not capture the dynamics of sign usage that characterizes digital communication. Hayles (2005) argued that

$\begin{array}{llllll}\text { Journal } & \text { of } & \text { Sociocybernetics } & 9 & \text { (2011), } & \text { pp. }\end{array}$


the disjunctions between Saussure's theory and the materially determined practices of code raise the question of whether it makes sense to use such legacy terms as "signifier" and "signified" with code. According to the American literary critic, theorists concerned with digital textuality tend to drift to frameworks that do not rely on these traditional terms.

Instead of signifier and signified, Hayles suggested Friedrich Kittler's argument to consider them as voltages. The signifieds are then the interpretations that other layers of code give to these voltages, or to say it another way, voltages at machine level would function as signifiers for a higher level to interpret them, and these interpretations in turn become signifiers for a still higher level interfacing them. Hayles (2005) stressed that the different levels of code consist of interlocking chains of signifiers and signifieds, with signifieds on one level becoming signifiers on another. Because all these operations depend on the ability of the machine to recognize the difference between one and zero, Saussure's premise that differences between signs make signification possible fits well with computer architecture.

Kittler (1999) wrote extensively about this epistemological breakdown. Commenting on Turing's projections toward programmers and mathematicians, the German media theorist thought that what disappeared in the split-up of binaries was not only the continuity of graphs and trajectories examined since Leibniz, but the difference between numbers and operational symbols, data and commands. For Kittler, even if numbers could be translated solely as data relationships, the signs + or - were still inhabited by human spirits who would understand them as addition or subtraction. Turing's Universal Discrete Machine, however, converted these letters into rows of binaries, and the machine language understands the command "add" neither as a human enunciation nor as a letter symbol, but just one of many series of bits. When commands, axioms, and sentences were converted into numbers, they also became manipulatable as numbers. The end of literary sentences would be an outcome of computers, whose endless series of numbers would decide only by the relative position whether they operate as verbal commands, numeric data or merely as addresses.

Hayles (2005) took Kittler's insight to think about the properties of code. At the level of binary code, the system can tolerate little if any ambiguity. While any physically embodied system accepts the presence of some noise, and thus some possible ambiguities, digital computers fix the noise within the system into unambiguous signals of one and zero before releasing it to the bitstream. As the system builds up levels of programming 
languages, it develops functionalities that permit increasingly greater ambiguities in the choices permitted or tolerated. Yet, according to Hayles (2005), it makes no sense to talk about signifiers without signifieds in regard to code, since every voltage change must have a precise meaning in order to affect the behavior of the machine. Every change at one level of programming code must be accurately correlated with another change at the other levels, or the machine will not be able to read it. For a machine, obsolete code is not a valid utterance. Even though this dynamic necessarily happens before (or after) any human interpretation of these messages, it nonetheless determines the constitution of every digital text.

The spectral sign represents a sign without actual reference, or to use Kittler's vocabulary, a "deanimalizated" sign that affects the core of the linguistic semiosis. The lack of a clear referent is a direct result of the saturation of semiotic circles and the atopic function of context in digital interactions. The practical application of this linguistic regimen is the capacity of a sign to refer not to another sign, but rather to an entire new sphere of meaning. This can be observed during the process of converting writing into code. The sign is corrupted in a process that is not related to language, but to interaction, which explains how the crosstalk (Shank 1993) emerges as a key feature in digital communication. It also explains how strictly linguistic and semiotic approaches fail to address the problem, as it transcends the sign as a closed system of differences (Saussure 1984), a fixed and limited network of dependencies (Hjelmslev 1961) or a consensual encyclopedia (Eco 1984).

The spectral sign emerges in view of the internal and recurring contradictions between the initial purpose of the utterance and the meanings conveyed throughout the communication process. Spectrality is therefore a semantic effect that cannot be detached from digital technology. The phenomenon can be seen in chat rooms and social networks, but also in blog comment areas that range from indifferent to cheerful and hostile within a minimum number of messages. In fact, when users retweet messages or commenters post to a blog from another blog, they create new contexts for the content and provide new meanings for the entries. When users subscribe to a given friend's feeds, they are aware the system will supply them a number of links culled from all over the web. Each link will have simultaneously two meanings: one related to the source, and the other relocated to the receiver. The process of relocation is what we addressed in this paper as spectrality.

$\begin{array}{lllllll}\text { Journal } & \text { of } & \text { Sociocybernetics } & 9 & \text { (2011), } & p p . & 3-17 \\ 15 & & & & \end{array}$




\section{References}

Aarseth, E. (1998) 'Aporia and Epiphany in Doom and The Speaking Clock' in Ryan, M.-L., ed. Cyberspace Textuality - computer technology and literary theory, Bloomington: Indiana University Press.

Barthes, R. (1957) Mythologies, Paris: Seuil.

Crystal, D. (2001) Language and the Internet, Cambridge: University Press.

Duke, R. D. (1974) Gaming: The Future's Language, New York: John Wiley \& Sons.

Eco, U. (1984) Semiotics and the philosophy of language, Bloomington: Indiana University Press.

Flusser, V. (2002) Writings, Minneapolis: University of Minnesota Press.

Hayles, K. (2005) My Mother was a Computer: Digital Subjects and Literary Texts, Chicago: University of Chicago Press.

Hjelmslev, L. (1961) Prolegomena to a Theory of Language, Madison: University of Wisconsin Press.

Kittler, F. (1999) Grammophon Film Typewriter, Stanford: Stanford University Press.

Mallarmé, S. and Weinfield, H. (1994) Collected poems, Berkeley: University of California Press. 
Panofsky, E. (1982) Meaning in the visual arts, Phoenix ed., Chicago: University of Chicago Press.

Prieto, L. J. (1966) Messages et Signaux, 1ํㅡㄹ. ed., Paris: Presses Universitaires de France.

Rothstein, A. (2009) 'Of 140-character-matology'. Accessed 15/04/2009.

Saussure, F. (1984) Cours de Linguistique Générale, Paris: Payot.

Shank, G. (1993) 'Abductive multiloguing: the semiotic dynamics of navigating the net', The Arachnet Electronic Journal on Virtual Culture, (1).

Shannon, C. E. (1949) 'Communication in the presence of noise', Proceeding of the institute of radio engineers, (37), 10-21.

Silverman, K. (1983) The subject of semiotics, New York: Oxford University Press.

Terranova, T. (2004) 'Communication beyond Meaning: On the Cultural Politics of Information', Social Text, 22(3), 51-73. 